\title{
A Clinico-Pathological Study of Gastric Polyps and Their Relationship with Helicobacter Pylori Infection atthe Pathology Departments of Hospitals in Yazd from 2011 To 2016
}

\author{
ShokouhTaghipour zahir ${ }^{1}$ Ebrahim Mahmooditabar ${ }^{2}$ Koorosh $_{\text {Rahmani }}{ }^{3}$ \\ Department of pathology, Faculty of medicine, ShahidSadoughi University of Medical sciences, Yazd, Iran \\ ${ }^{I}$ Department of pathology, ShahidSadoughi University of Medical sciences, Yazd, Iran \\ ${ }^{2}$ Department of pathology, ShahidSadoughi University of Medical sciences, Yazd, Iran \\ ${ }^{3}$ Department of pathology, ShahidSadoughi University of Medical sciences, Yazd, Iran
}

\begin{abstract}
:
Background: Gastric polyps are gastric lesions with a relatively low prevalence that are mostly asymptomatic and detected incidentally by endoscopy. Since some polyps are associated with Helicobacter pylori infection and can be a precursor to future malignancies, this research carries out a clinicopathological study of gastric polyps in hospitals in Yazd, Iran.

Materials and Methods: The records of all the patients who had undergone endoscopy from 2011 to 2016 and had pathology reports of gastric polyps were extracted from the archives of the hospitals' pathology departments and their demographic details, including age, gender, the anatomic site of polyp, clinical symptoms, Helicobacter pylori infection and the histopathology of the polyp were examined and recorded in a pre-developed checklist. The data obtained were analyzed in SPSS-17 at the significance level of $P<0.05$.

Results: Of the 72 patients studied, 25 (34.7\%) were male and 47 (65.3\%) were female. Polyps were mostly prevalent in the over-60 age group $(n=33,45.8 \%)$. The most common anatomic site of the extracted polyps was the body of the stomach $(n=38,52.8 \%)$. Abdominal pain was the most frequent clinical complaint in the patients $(n=37,51.4 \%)$, and hyperplastic polyps were also the most frequent type of polyps observed ( $n=46,63.9 \%)$. Most of the polyps had no concomitant pathologies (n=41, 56.9\%). Only in 12 cases $H$. pylori infection was observed, nine of whom happened to be female. H. pylori infection was observed mostly in the age group of 45 60. No significant correlation was found between gender and H. Pylori infection in gastric polyps.

Conclusion: In line with the results of previous studies on the subject, the incidence of polyps increases with age, and since most polyps are hyperplastic, their malignancy is not very likely. Nonetheless, people with idiopathic abdominal pain should be examined for gastric polyps.
\end{abstract}

Keywords: Gastric, polyp, Helicobacter pylori, infection

\section{Introduction}

Polyp is a visible protrusion from the mucosal surface. Epithelial polyps are the most common benign gastric tumors that often develop in the gastric antrum [1]. The prevalence of gastric polyps varies from $0.8 \%$ to $2.4 \%$ in the general population and includes mainly adenomatous, hyperplastic, hamartomatous, inflammatory and heterotopic (ectopic pancreas) types of polyp[2]. The most common gastric polyp is hyperplastic polyp (75\%), which is often benign and in multiple form and mainly develops under chronic inflammatory conditions and is less likely to become malignant [2,3]. Polyps may regress, remain constant or enlarge over time; however, these polyps often regress after the eradication of the $\mathrm{H}$. pylori infection $[2,4]$. Men and women are equally affected and mainly in mid to late adulthood. Adenomatous polyps may cause malignant changes, but very few of these lesions ultimately become malignant. H. pylori is a gram-negative, curved, flagellated bacillus that is found only in the epithelium or metaplastic epithelium of the stomach and mostly settles in the innermost mucosal gel of the stomach mucosal lining. This bacterium first spreads in the gastric antrum and then migrates to more proximal parts of the stomach over time. Helicobacter pylori (H. pylori) is the most common cause of bacterial infection in the world. Its prevalence is associated significantly with age and varies from $10 \%$ in people below age 30 to $60 \%$ in those over age $60[4,5]$. This infection is more prevalent in developing countries and affects more than $80 \%$ of the population by age 20 . H. pylori infection is always caused in conjunction with a chronic active gastritis. H. pylori infection can cause gastritis, peptic ulcer, mucosal associated lymphoid tumor (MALT) lymphoma and stomach cancer $[1,3,6]$. The location of gastric polyps has changes overtime fromantrum to corpus [7]. However, most of gastric polyps were according to previous studies, gastric polyps are associated with $\mathrm{H}$. pylori, and according to published articles, half of hyperplastic gastric polyps are associated with H. pylori infection. Since hyperplastic gastric polyps are often developed with an underlying gastritis and since $\mathrm{H}$. pylori infection causes gastritis, the present study was conducted to examine the gastric 
polyps detected in hospitals in terms of anatomic site, clinical symptoms, types and concomitant H. pylori infection.

\section{Material And Methods}

The present retrospective, descriptive, analytical study was conducted using convenience sampling. After obtaining permission from the ethics committee of the School of Medicine and visiting the hospital pathology departments of Yazd, all the patients who had presented to these departments from 2011 to 2016 and had pathology records of gastric polyps were entered into the study. Pathology slides were extracted from the archives and re-examined by two pathologists for their type. The patients were examined according to a predeveloped checklist inquiring about variables such as age, gender, year of biopsy, endoscopic results, biopsy results (type of polyp), presence or absence of $\mathrm{H}$. pylori infection, anatomic site of polyp, concomitant pathologies (such as intestinal metaplasia, acute and chronic gastritis, H. pylori infection, polyps in other sites and celiac disease) and clinical symptoms (abdominal pain, vomiting, dysphagia, diarrhea, anemic reflux, gastrointestinal bleeding, history of polyp and history of surgery). The data obtained were entered into the checklist and analyzed in SPSS-17 using the Chi-square test and Fisher's exact test at the significance level of $\mathrm{P}<0.05$.

\section{Results}

This study was conducted on 72 patients who had presented to the pathology departments of hospitals affiliated to ShahidSadoughi University of Medical Sciences in Yazd from 2011 to 2016; 25 (34.7\%) of the patients were male and $47(65.3 \%)$ were female. According to Table 1, most of the patients were in the over-60 age group $(45.8 \%)$. The most frequent anatomic site affected was the body of the stomach $(\mathrm{n}=38,52.8 \%)$ and the least frequent site was the pyloric region $(n=1,1.4 \%)$. The most common clinical symptoms $(n=37,51.4 \%)$ included abdominal pain, vomiting, dysphagia, diarrhea and reflux (Table 2). In terms of type, the most frequent polyps were hyperplastic $(n=46,63.9 \%)$, and the least frequent were angiomatous and hamartomatous types (1.4\% for each type; Table 1$)$. A total of $41(56.9 \%)$ of the polyps had no concomitant pathologies (Table 3$)$. In the patients with hyperplastic polyps, the most frequent clinical symptom was epigastric pain $(n=22,47.8 \%)$, followed by melena and lower gastrointestinal bleeding $(\mathrm{n}=10,21.8 \%)$. The least frequent clinical symptom was upper gastrointestinal bleeding $(n=1,2.2 \%)$. The most common type of gastric polyp found was hyperplastic both in men $(n=16,34.8 \%)$ and in women $(n=30,65.2 \%$; Table 4). H. Pylori was detected in twelve out of 72 polyps, $75 \%(n=9)$ in women and $25 \%(n=3)$ in men. No significant correlation was found between gender and H. Pylori infection in gastric polyps.

\section{Discussion}

This study was conducted on 72 patients diagnosed with gastric polyps over a five-year period. Most of the patients who had polyps were female in this study. Similarly, in a study conducted by Cao et al., the incidence of polyp was higher in women than in men [8]. Most polyps in their study were fundus gland polyps (FGPs) and polyps and gender were therefore found to be related; but in the present study, despite the higher prevalence of the female patients, no significant relationship were observed between polyps and gender. The disparity between these two studies may be due to the small sample size used in the present study [8].Most polyps in the present study were in the body of the stomach, which is consistent with the results obtained by Markowski et al., although researchers used to argue that most gastric polyps develop in the antral region [8,9] The most frequent age group affected in this study was the over-60 age group, which is inconsistent with the results obtained by Fan N-N et al .(2015) who had found the 45 to 60 age group to be most affected by polyps and had reported the incidence of gastric polyps to reduce after age 60; in the present study, however, the incidence of polyps increased with age [7]. H. pylori infection was three times more prevalent in women than in men, although no significant relationships were observed between gender and the type of polyp. A review of literature shows that, hyperplastic polyps were the most common type of polyp concomitant with H. pylori infection $[3,4,6]$ In line with other studies, most polyps had no concomitant pathologies in the present study. In a study conducted by Markowski AR et al on the clinical symptoms of polyps, most polyps were found to be asymptomatic and were detected incidentally, also in the present study, the most frequent clinical symptom reported was abdominal pain [9]. The disparity between the results of the present study and other studies may be attributed to the time factor, i.e. the duration of time since the development of the polyp, as most polyps begin to show clinical symptoms a long time after their incidence, while in hospitals that offer endoscopy services, polyps are detected when they are smaller and still asymptomatic [8, 9]. In the present study, the age group mostly infected with $\mathrm{H}$. pylori was the 45 to 60 age group. In the study by Islam et al., the most frequent clinical symptoms associated with hyperplastic polyps were dyspepsia, heartburn and upper gastrointestinal bleeding, and although these symptoms had a relatively high prevalence in the present study, no significant relationships were observed between the type of polyp and clinical symptoms in this research [10]. Hyperplastic polyps can 
be singular or multiple and can present independently of other conditions or concomitant with familial polyposis syndrome [6,7]. In the present study, hyperplastic polyps made up $69.3 \%$ of all the reported gastric polyps, which agrees with the results obtained by Markowski AR et al, who reported that, although most gastric polyps in the US are of the fundus type, $25 \%$ to $71 \%$ of the polyps found in their study were hyperplastic [9]. The incidence of hyperplastic polyps increases with age and they are mostly observed in people over $65[1,4]$. These findings are consistent with the present findings regarding the higher prevalence of polyps in the age group over 60 and the highest frequency of polyp type pertaining to hyperplastic polyps. Studies have reported a relationship between $\mathrm{H}$. pylori infection and hyperplastic polyps, although no significant relationships were observed in the present study between the type of polyp detected and $\mathrm{H}$. pylori infection, which may be due to the small sample size assessed. Most gastric polyps are detected incidentally in endoscopic examinations performed for other reasons and are mostly asymptomatic. In the endoscopic view, some polyps are in plaque form, others are in a nodular or lobular form and a few are attached without a stalk [7, 9]. Most patients examined in the present study had presented with clinical symptoms such as abdominal pain, vomiting, dysphagia, diarrhea and reflux, which is inconsistent with the results of similar studies. In terms of type, gastric polyps do not differ greatly from each other in the endoscopic view and their differentiation thus requires a histopathological examination. The anatomic site of polyps can, however, guide the endoscopist, as adenomatous polyps are mostly found in the antral region while fundus type polyps are not [5, 7]. Gastric polyps are divided into epithelial, hamartomatous, and mesenchymal categories based on their pathologic view. In the present study, the patients' polyps had been removed only as gastric polyps in an endoscopic examination without having their type reported,and only the pathology report had differentiated between them.

\section{Conclusions}

In line with the results of previous studies on the subject, the incidence of polyps increases with age, and sincemost polyps are hyperplastic, their malignancy is not very likely. Nonetheless, people with idiopathic abdominal pain should be examined for gastric polyps. H. pylori infection does not appear to be greatly responsible for gastric polyps.

\section{References}

[1]. Genta RM, Sonnenberg A. Characteristics of the Gastric Mucosa in Patients With Intestinal Metaplasia. Am J SurgPathol. 2015 May;39(5):700-704.

[2]. Sonnenberg A, Genta RM. Prevalence of benign gastric polyps in a large pathology database. Dig Liver Dis. 2015 Feb;47(2):164-9.

[3]. Albuquerque A, Rios E, Carneiro F, Macedo G. Evaluation of clinico-pathological features and Helicobacter pylori infection in gastric inflammatory fibroid polyps. Virchows Arch. 2014 Dec;465(6):643-7.

[4]. Huang CZ, Lai RX, Mai L, Zhou HL, Chen HJ, Guo HX. Relative risk factors associated with the development of fundic gland polyps. Eur J GastroenterolHepatol. 2014 Nov;26(11):1217-21.

[5]. Samarasam I, Roberts-Thomson J, Brockwell D. Gastric fundic gland polyps: a clinico-pathological study from North West Tasmania. ANZ J Surg. 2009 Jun;79(6):467-70.

[6]. Tokunaga K1, Tanaka A, Takahashi S. [Gastric hyperplastic polyps and H. pylori infection, their relationship and effects of eradication therapy]. Nihon Rinsho. 2013 Aug;71(8):1449-52.

[7]. Fan NN, Yang J, Sun G, Lu ZS, Ling Hu EQ, Wang XD, et al. Changes in the spectrum of gastric polyps in the Chinese population.World J Gastroenterol. 2015 Sep 7;21(33):9758-64.

[8]. Cao H, Wang B, Zhang Z, Zhang H, Qu R. Distribution trends of gastric polyps: an endoscopy database analysis of 24121 northern Chinese patients. - J GastroenterolHepatol. 2012 Jul;27(7):1175-

[9]. Markowski AR, Markowska A, Guzinska-Ustymowicz K. Pathophysiological and clinical aspects of gastric hyperplastic polyps. World J Gastroenterol. 2016 Oct 28;22(40):8883-8891.

[10]. Islam RS, Patel NC, Lam-Himlin D, Nguyen CC. Gastric polyps: a review of clinical, endoscopic, and histopathologic features and management decisions. GastroenterolHepatol (N Y). 2013 Oct;9(10):640-51.

Table1: Demographic characteristics of patients with gastric polyps

\begin{tabular}{|l|l|}
\hline & Frequency N\% \\
\hline Gender & \\
\hline Male & $25(34.7 \%)$ \\
\hline Female & $47(65.3 \%)$ \\
\hline Age group & \\
\hline$<45$ & $13(18.1 \%)$ \\
\hline $45-60$ & $26(36 \%)$ \\
\hline$>60$ & $33(45.8 \%)$ \\
\hline Anatomical site of polyps & \\
\hline Body & $38(52.8 \%)$ \\
\hline antrum & $18(25 \%)$ \\
\hline Fundus & $13(18.1 \%)$ \\
\hline cardia & $2(2.8 \%)$ \\
\hline Pylorus & $1(1.4 \%)$ \\
\hline Polyp type & \\
\hline Hyperplastic & $46(63.9 \%)$ \\
\hline inflammatory & $16(22.2 \%)$ \\
\hline
\end{tabular}




\begin{tabular}{|l|l|}
\hline Adenomatous & $8(11.1 \%)$ \\
\hline Angiomatous & $1(1.4 \%)$ \\
\hline hamartomatous & $1(1.4 \%)$ \\
\hline Helicobacter pylori infection & \\
\hline Male & $3(25 \%)$ \\
\hline Female & $9(75 \%)$ \\
\hline Total & $72(100 \%)$ \\
\hline
\end{tabular}

Table 2: Clinical characteristics of patients with gastric polyps

\begin{tabular}{|l|l|l|}
\hline Related Clinical history & Frequency $(\mathbf{N})$ & \% \\
\hline Abdominal pain, dysphagia, reflux & 37 & 51.4 \\
\hline Anemia and melena & 11 & 15.3 \\
\hline Previous Polyp or surgery & 5 & 6.9 \\
\hline Without clinical symptoms & 19 & 26.4 \\
\hline Total & 72 & 100 \\
\hline
\end{tabular}

Table 3: Pathology findings in gastric polyps

\begin{tabular}{|l|l|l|}
\hline Concomitant pathology & Frequency $(\mathbf{N})$ & \% \\
\hline Active and chronic gastritis & 16 & 22.2 \\
\hline H. pylori infection & 12 & 16.7 \\
\hline Additional polyps in other areas of GI & 2 & 2.8 \\
\hline Celiac disease & 1 & 1.4 \\
\hline Without any other pathology & 41 & 56.9 \\
\hline Total & 72 & 100 \\
\hline
\end{tabular}

Table 4: Frequency distribution of gastric polyps based on gender

\begin{tabular}{|l|l|l|l|}
\hline Polyp Type & Male N(\%) & Female N(\%) & Total \\
\hline Hyperplastic & $16(34.8)$ & $30(65.2$ & 46 \\
\hline Inflammatory & 6 & $10(62.5$ & 16 \\
\hline Adenomatous & 2 & $6(75$ & 8 \\
\hline Angiomatous & 1 & $0(0$ & 1 \\
\hline Hamartomatous & 0 & $1(100)$ & 1 \\
\hline Total & $25(34.7)$ & $47(65.3)$ & 72 \\
\hline
\end{tabular}

